\title{
The ketogenic diet as a treatment paradigm for diverse neurological disorders
}

\author{
Carl E. Stafstrom ${ }^{1,2}$ and Jong M. Rho ${ }^{3,4}$ * \\ ${ }^{1}$ Department of Neurology, University of Wisconsin, Madison, WI, USA \\ ${ }^{2}$ Department of Pediatrics, University of Wisconsin, Madison, WI, USA \\ ${ }^{3}$ Department of Pediatrics, University of Calgary Faculty of Medicine, Calgary, AB, Canada \\ ${ }^{4}$ Department of Clinical Neurosciences, University of Calgary Faculty of Medicine, Calgary, AB, Canada
}

Edited by:

Yuri Zilberter, INSERM U751, Faculté

de Médecine Timone, France

Reviewed by:

Yuri Zilberter, INSERM U751, Faculté

de Médecine Timone, France

Marta Balietti, Istituto Nazionale di

Ricovero e Cura per Anziani, Italy

*Correspondence:

Jong M. Rho, Alberta Children's

Hospital, University of Calgary, 2888

Shaganappi Trail Northwest, Calgary,

AB, Canada T3B 6 A8.

e-mail:jmrho@ucalgary.ca
Dietary and metabolic therapies have been attempted in a wide variety of neurological diseases, including epilepsy, headache, neurotrauma, Alzheimer disease, Parkinson disease, sleep disorders, brain cancer, autism, pain, and multiple sclerosis. The impetus for using various diets to treat - or at least ameliorate symptoms of - these disorders stems from both a lack of effectiveness of pharmacological therapies, and also the intrinsic appeal of implementing a more "natural" treatment. The enormous spectrum of pathophysiological mechanisms underlying the aforementioned diseases would suggest a degree of complexity that cannot be impacted universally by any single dietary treatment. Yet, it is conceivable that alterations in certain dietary constituents could affect the course and impact the outcome of these brain disorders. Further, it is possible that a final common neurometabolic pathway might be influenced by a variety of dietary interventions. The most notable example of a dietary treatment with proven efficacy against a neurological condition is the high-fat, low-carbohydrate ketogenic diet (KD) used in patients with medically intractable epilepsy. While the mechanisms through which the KD works remain unclear, there is now compelling evidence that its efficacy is likely related to the normalization of aberrant energy metabolism. The concept that many neurological conditions are linked pathophysiologically to energy dysregulation could well provide a common research and experimental therapeutics platform, from which the course of several neurological diseases could be favorably influenced by dietary means. Here we provide an overview of studies using the KD in a wide panoply of neurologic disorders in which neuroprotection is an essential component.

Keywords: ketogenic diet, neuroplasticity, epilepsy, neurological disorders

\section{INTRODUCTION}

The ketogenic diet (KD) is now a proven therapy for drug-resistant epilepsy (Vining et al., 1998; Neal et al., 2008), and while the mechanisms underlying its anticonvulsant effects remain incompletely understood (Hartman et al., 2007; Bough and Stafstrom, 2010; Rho and Stafstrom, 2011), there is mounting experimental evidence for its broad neuroprotective properties and in turn, emerging data supporting its use in multiple neurological disease states (Baranano and Hartman, 2008). Even in patients with medically refractory epilepsy who have remained seizure-free on the KD for 2 years or more, it is not uncommon for clinicians to observe that both anticonvulsant medications and the diet can be successfully discontinued without recrudescence of seizures (Freeman et al., 2007). This intriguing clinical observation forms the basis of the hypothesis that the KD may possess anti-epileptogenic properties.

This review article explores the rationale for using the KD and related dietary treatments in neurological disorders outside of epilepsy, and summarizes the clinical experience to date. An underlying theme of such diet-based therapies is that nutrients and metabolic substrates can exert profound effects on neuronal plasticity, modifying neural circuits and cellular properties to enhance and normalize function. At a fundamental level, any disease in which the pathogenesis is influenced by abnormalities in cellular energy utilization - and this implies almost every known condition - would theoretically be amenable to the KD. It is important to acknowledge that much of the data discussed here are preliminary and anecdotal, and hence need to be validated by well-controlled prospective studies. Nevertheless, that diet and nutrition should influence brain function should not be altogether surprising, and there are already abundant clinical and laboratory data linking defects in energy metabolism to a wide variety of disease states (Waldbaum and Patel, 2010; Roth et al., 2011; Schiff et al., 2011). Thus, the potential for interesting and novel applications of the $\mathrm{KD}$ and related dietary therapies is almost limitless (Stafstrom, 2004).

\section{NEUROPROTECTIVE ROLE OF THE KD}

Over the past decade, investigators have identified numerous mechanisms through which the KD may provide neuroprotective activity. While a comprehensive discussion of such mechanisms is beyond the scope of this chapter, a brief discussion is warranted as such actions are intimately related to disorders that share the common feature of progressive neurodegeneration and/or cellular 
bioenergetic dysfunction. The reader is referred to recent reviews for more details on this subject (Gasior et al., 2006; Acharya et al., 2008; Masino and Geiger, 2008).

Two hallmark features of KD treatment are the rise in ketone body production by the liver and a reduction in blood glucose levels. The elevation of ketones is largely a consequence of fatty acid oxidation. Specific polyunsaturated fatty acids (PUFAs) such as arachidonic acid, docosahexaenoic acid, and eicosapentaenoic acid, might themselves regulate neuronal membrane excitability by blocking voltage-gated sodium and calcium channels (Voskuyl and Vreugdenhil, 2001), reducing inflammation through activation of peroxisome proliferator-activated receptors (PPARs; Cullingford, 2008; Jeong et al., 2011), or inducing expression of mitochondrial uncoupling proteins which reduce reactive oxygen species (ROS) production (Bough et al., 2006; Kim do and Rho, 2008). Ketone bodies themselves have been shown to possess neuroprotective properties, by raising ATP levels and reducing ROS production through enhanced NADH oxidation and inhibition of mitochondrial permeability transition (mPT; Kim do et al., 2007). Along similar lines of improved bioenergetics, the KD has been shown to stimulate mitochondrial biogenesis, resulting in stabilized synaptic function (Bough et al., 2006).

The second major biochemical feature of the KD is the decrease in glycolytic flux. Reduction of glycolysis is an essential feature of calorie restriction, which has been shown to suppress seizures (Greene et al., 2001) as well as prolong the lifespan of numerous species, including primates (Kemnitz, 2011; Redman and Ravussin, 2011). While the link between calorie restriction and KD mechanisms remain controversial (Yamada, 2008; Maalouf et al., 2009), it is clear that both treatments result in reduction of blood glucose, likely involving reduced glycolytic flux. In that regard, 2-deoxy-Dglucose (2DG), an analog of glucose that blocks phosphoglucose isomerase and hence inhibits glycolysis, has been shown to block epileptogenesis in the rat kindling model by decreasing the expression of brain-derived neurotrophic factor (BDNF) and its principal receptor, tyrosine kinase B (TrkB; Garriga-Canut et al., 2006). Several other important mechanisms contribute to the neuroprotective consequences of calorie restriction, including improved mitochondrial function and decreased oxidative stress (similar to that seen with ketones and PUFAs), decreased activity of proapoptotic factors, and inhibition of inflammatory mediators such as interleukins and tumor necrosis factor alpha (TNF $\alpha$; Maalouf et al., 2009).

In the end, there are likely many other mechanisms that could contribute to the neuroprotective properties of the KD. Many of these mechanisms are thought to relate principally to the KD's anticonvulsant effects, but some if not all of them could contribute to cellular homeostasis and preventing neuronal injury or dysfunction. An important caveat, however, is that yet unidentified mechanisms may operate in diseases outside of epilepsy, and this possibility presents further opportunities for examining the pleiotropic effects of this metabolism-based therapy at a mechanistic level.

\section{THE KD IN EPILEPSY}

There is no longer any doubt that the $\mathrm{KD}$ is effective in ameliorating seizures in patients, especially children, with medically refractory epilepsy (Vining, 1999; Neal et al., 2008; Freeman et al., 2009). After its introduction in 1920, the KD was used as a first or second-line treatment for severe childhood epilepsy. With the introduction of anticonvulsant medications in convenient pill form, the use of the KD waned, only to resurge later in the early 1990s, due largely to the efforts of concerned parents who brought the diet back to greater public awareness (Wheless, 2008). Recent years have witnessed a remarkable surge in research on the $\mathrm{KD}$, including basic science efforts as well as clinical protocols and trials (Kim do and Rho, 2008; Neal et al., 2008; Kessler et al., 2011). The KD has now become an integral part of the armamentarium of most major epilepsy centers throughout the world (Kossoff and McGrogan, 2005).

\section{THE KD IN AGING}

Aging involves the gradual decrease in function, and at times outright degeneration, of neurons and neural circuits. It is possible that by altering energy metabolism with the KD, rates of degeneration of certain neural structures and functions might be slowed (Balietti et al., 2010a). However, KDs may induce differential morphological effects in structures such as the hippocampus, perhaps as a consequence of region-specific neuronal vulnerability during the late aging process (Balietti et al., 2008). Specifically, it has been shown that the medium-chain triglyceride (MCT) form of the KD may induce detrimental synaptic changes in CA1 stratum moleculare, but beneficial effects in the outer molecular layer of the dentate gyrus (Balietti et al., 2008). In MCT-fed aged rats compared to aged rats receiving a normal diet, mitochondrial density and function in cerebellar Purkinje cells were significantly increased, suggesting that the $\mathrm{KD}$ can rescue age-related mitochondrial dysfunction (Balietti et al., 2010b). These observations imply certain risks, but also potential benefits of the KD for the aging brain. However, the fact that the KD reduces oxidative stress and its downstream consequences provides a reasonable rationale for considering this type of treatment to retard the adverse consequences during aging (Freemantle et al., 2009). As an example, T-maze and object recognition performance were improved in aged rats by $\mathrm{KD}$ administration, suggesting a potential functional benefit in cognition (Xu et al., 2010). Finally, it should be noted that because of its similarities to calorie restriction (as noted above), the KD is likely to involve other neuroprotective mechanisms that could ameliorate pathological aging - especially when occurring in the context of neurodegeneration (Contestabile, 2009).

\section{THE KD IN ALZHEIMER DISEASE}

There is growing realization that neuronal excitability is enhanced in patients with Alzheimer disease (AD; Noebels, 2011; Roberson et al., 2011). While the essential pathological processes of $\mathrm{AD}$ involves neuronal degeneration with accumulation of abnormal cellular products such as fibrillary plaques and tangles, recent evidence points to alterations in the function of extant neural circuits and mitochondrial homeostasis (Kapogiannis and Mattson, 2011). This view is bolstered by the higher incidence of seizures in patients with $\mathrm{AD}$ as compared to the unaffected population (Palop and Mucke, 2009). Therefore, there is a rationale for hypothesizing that the KD might have a beneficial role in patients with 
$\mathrm{AD}$ (Balietti et al., 2010a), in addition to the potential benefits to the aging process as noted above. One should note, importantly, that if ketone bodies are indeed the primary mediators that counter aging and neurodegeneration in $\mathrm{AD}$, implementation of the KD should be tempered by known age-related differences in the production and extraction of ketones (i.e., this is more efficient in young animals), as well as age-specific regional differences in ketone utilization within the brain (Nehlig, 1999).

Clinical studies to date have been equivocal but promising. A randomized double-blind, placebo-controlled trial of a MCT $\mathrm{KD}$ resulted in significantly improved cognitive functioning in $\mathrm{APO} \varepsilon 4$-negative patients with $\mathrm{AD}$ but not in patients with a $\mathrm{APO} \varepsilon 4$ mutation (Henderson et al., 2009). In this study, the primary cognitive end-points measured were the mean change from baseline in the AD Assessment Scale-Cognitive subscale, and global scores in the AD Cooperative Study - Clinical Global Impression of Change (Henderson et al., 2009). This significant clinical improvement was considered to be secondary to improved mitochondrial function, since ketone bodies (specifically, beta-hydroxybutyrate or $\mathrm{BHB}$ ) have been shown to protect against the toxic effects of $\beta$-amyloid on neurons in culture (Kashiwaya et al., 2000). Alternatively, the KD may actually decrease amounts of $\beta$-amyloid deposition (VanderAuwera et al., 2005). Interestingly, other diets such as the Mediterranean diet are showing some promise in $\mathrm{AD}$ ( $\mathrm{Gu}$ et al., 2010), possibly through a reduction in systemic inflammation and improved metabolic profiles.

Recent studies have shown a closer linkage of AD to epilepsy. For example, animal models of $\mathrm{AD}$ exhibit neuronal hyperexcitability and enhanced propensity to seizures (Palop et al., 2007; Roberson et al., 2011); these models may ultimately allow for detailed analyses of both cognitive and anticonvulsant effects of the KD or other dietary manipulations such as calorie restriction. Transgenic AD mice fed 2DG demonstrated better mitochondrial function, less oxidative stress, and reduced expression of amyloid precursor protein and $\beta$-amyloid compared to control animals (Yao et al., 2011).

Another pathophysiological mechanism hypothesized to operate in $\mathrm{AD}$ ties together altered mitochondrial function and glucose metabolism, i.e., accumulation of advanced glycation endproducts (AGE; Srikanth et al., 2011). AGE accumulation is a process of normal aging that is accelerated in $\mathrm{AD}$; proteins are non-enzymatically glycosylated and this cross-linking of proteins accentuates their dysfunction. One proposed mechanism is increased ROS and free radical formation, which, as discussed above, hampers mitochondrial function. The intriguing possibility that AGE inhibitors (e.g., aminoguanidine, tenilsetam, carnosine) could act in concert with the $\mathrm{KD}$ or antioxidants in retarding $\mathrm{AD}$ progression remains speculative at this time.

Thus, there is growing evidence that the KD may be an effective treatment for $\mathrm{AD}$ through a variety of metabolism-induced mechanisms that reduce oxidative stress and neuroinflammation, and enhance bioenergetic profiles - largely through enhanced mitochondrial functioning. However, caution should be exercised in extrapolating findings in animals to humans, as discrepancies in terms of both clinical efficacy and untoward side-effects have been noted. For example, adverse reactions to calorie restriction have been reported in some rodent models (Maalouf et al., 2009), and in hippocampus, abnormal morphological synaptic changes have been observed in CA1 stratum moleculare (Balietti et al., 2008).

\section{THE KD IN PARKINSON DISEASE}

The primary pathophysiology in Parkinson disease (PD) is excitotoxic degeneration of dopaminergic neurons in the substantia nigra, leading to abnormalities of movement, and to an increasing extent, in cognition and other cortical functions. How could the KD benefit patients with PD? Based on the recognition that ketone bodies may bypass defects in mitochondrial complex I activity that have been implicated in PD, a small clinical study demonstrated that 5 of 7 affected patients showed improved scores on a standard PD rating scale (Vanitallie et al., 2005); however, given the small sample size, a placebo effect cannot be ruled out. In animal models of PD produced by 1-methyl-4-phenyl-1,2,3,6tetrahydropyridine (MPTP), BHB administration ameliorated the mitochondrial respiratory chain damage that ordinarily results from that toxin (Kashiwaya et al., 2000). Additional evidence supporting the potential benefits of ketone bodies in PD is provided by in vitro experiments demonstrating the protective effects of these substrates against mitochondrial respiratory chain dysfunction induced exogenously by complex I and II inhibitors rotenone and 3-nitropropionic acid, respectively (Kim do et al., 2010), and even anti-inflammatory actions of the KD on MPTP-induced neurotoxicity (Yang and Cheng, 2010). It would be of interest to determine whether commercially available treatments that augment ketonemia - e.g., the MCT-based formulation used in a recent Alzheimer's clinical trial (Henderson et al., 2009) - might benefit patients with $\mathrm{PD}$.

\section{THE KD IN AMYOTROPHIC LATERAL SCLEROSIS}

Amyotrophic lateral sclerosis (ALS) is a rapidly progressive disease due to degeneration of motor neurons of the cortex and anterior horn of the spinal cord. As a consequence, voluntary motor activity gradually deteriorates, leaving the affected individual profoundly weak despite largely retained cognitive functioning. The essential pathophysiological mechanisms that underlie this relentless disorder are yet to be fully elucidated, but similar to other neurodegenerative disorders, the involvement of energy-producing systems likely play a role and mitochondrial dysfunction probably contributes to disease pathogenesis. In this regard, the KD may be a promising adjunctive treatment for this devastating disease (Siva, 2006), as evidenced in a mouse model of ALS, produced by knocking out the gene encoding the copper/zinc superoxide dismutase SOD1-G93A, causing progressive muscle weakness and death by respiratory failure. Administration of a KD to these mutant mice led to both histological (higher motor neuron counts) and functional improvements (preserved motor function on the rotorod test) compared to non-KD fed animals (Zhao et al., 2006). However, the KD did not extend survival time compared to non-KD fed control mice. Mitochondria from these mutant mice demonstrated increased ATP synthesis, countering the inhibition of complex I of the electron transport chain. It is important to note that approximately $20 \%$ of the familial cases of ALS have SOD1 mutations, and hence the possibility arises that the KD may be of benefit to patients with ALS. 
One potentially important consideration in this regard-applicable to all neurodegenerative diseases - is determining whether timing of intervention is crucial for a protective effect by $\mathrm{KD}$ treatment. Neurological disorders in late stages of progression may have such extreme neuronal dysfunction and death to allow a "refueling" with metabolic substrates to help recover integrity and function. Certainly, this appears to be the case in a small pilot study of $\mathrm{KD}$ treatment in patients with Lafora body disease (Cardinali et al., 2006).

\section{THE KD IN CANCER}

Cells that exhibit the most active metabolic rates (i.e., cancer cells) are most sensitive to the lack of metabolic energy to fuel their activity, a well-recognized biochemical phenomenon known as the Warburg effect. Theoretically, depriving rapidly dividing, highly metabolic cancer cells of their usual fuel supply, e.g., glucose (by use of the KD or 2DG), could be clinically therapeutic (Aft et al., 2002; Pelicano et al., 2006; Otto et al., 2008). Despite this well documented cellular observation, the $\mathrm{KD}$ has only recently been considered as a clinical treatment in the oncology field.

Pioneering work by Seyfried et al. (2011) over the past decade has shown that animals with experimentally produced brain tumors placed on a KD exhibit markedly decreased tumor growth rates, and these remarkable effects appear to be a consequence of calorie restriction (i.e., reduced blood glucose levels) rather than KD-induced ketosis (i.e., fatty acid oxidation) as the principal mechanism. Other investigators have found similar effects of the $\mathrm{KD}$ in animal models. One group found that the KD reduces ROS production in malignant glioma cells, and gene microarray expression profiling demonstrated that the $\mathrm{KD}$ induces an overall reversion to patterns seen in non-tumor specimens and a reduction in the expression of genes encoding signal transduction pathways and growth factors known to be involved in glioma growth (Stafford et al., 2010). It is also interesting to note that PPAR $\alpha$-activated by nutrients such as fatty acids - is now a target for developing anti-cancer drugs that target mitochondrial metabolism (Grabacka et al., 2010).

While clinical validation of this phenomenon is not yet forthcoming, there are several case reports suggesting that the $\mathrm{KD}$ may be efficacious in humans with brain tumors. Nebeling et al. (1995) reported beneficial effects of an MCT-based diet in two pediatric patients with advanced stage malignant astrocytomas. More recently, Zuccoli et al. (2010) described a case study of an elderly woman with glioblastoma multiforme who was treated with standard radiotherapy plus concomitant temozolomide therapy together with a calorie-restricted $\mathrm{KD}$, and a complete absence of brain tumor tissue was noted on FDT-PET and MRI imaging after 2 months of treatment - results the authors attributed in part to the adjunctive dietary treatment. Further, in a pilot trial of the $\mathrm{KD}$ in 16 patients with advanced metastatic tumors, six individuals reported improved emotional functioning and less insomnia, indicating that in some instances, the KD may lead to improved quality of life (Schmidt et al., 2011). In contrast, a retrospective examination of five patients with tuberous sclerosis complex treated with the KD indicated either a lack of tumor suppression or further tumor growth (Chu-Shore et al., 2010). Thus, it may be that distinct tumor types within different organ systems may respond differently to the KD or other dietary treatments and that such differences may reflect variations in the metabolic vulnerability of specific tumor types, perhaps through intrinsic differences in the expression of metabolism-related genes (Stafford et al., 2010).

\section{THE KD IN STROKE}

To date, no clinical trials of the KD have been performed in patients with stroke, but several animal studies of hypoxia-ischemia support the potential beneficial effect of the diet. Most of these models entail pre-treatment with the $\mathrm{KD}$ (or with $\mathrm{BHB}$ ), resulting in decreased structural and functional damage from the stroke. For example, Tai et al. (2008) utilized a cardiac arrest model in rats and found significantly reduced Fluoro-Jade staining in animals that underwent 25 days of pre-treatment with the KD. These investigators later determined that these effects were not due to involvement of plasmalemmal ATP-sensitive potassium channels (Tai et al., 2009), which have been implicated in ketone body action (Ma et al., 2007). Other researchers have hypothesized that the neuroprotective properties of ketone bodies might be related to up-regulation of hypoxia inducible factor (HIF1- $\alpha$ ) which is important in angiogenesis and anti-apoptotic activity (Puchowicz et al., 2008). In that study, pre-treatment with BHB (via intraventricular infusion, followed by middle cerebral artery occlusion) led to significant increases in brain succinate content, as well as elevations in HIF1- $\alpha$ and $\mathrm{Bcl}-2$, an anti-apoptotic protein. To be clinically meaningful, of course, a positive effect must be demonstrable after, and not before, an ischemic event. Nevertheless, such studies imply that biochemical alterations that favor energy metabolism would be protective against acute forms of severe brain injury.

\section{THE KD IN MITOCHONDRIAL DISORDERS}

As mentioned above, given the growing evidence that the $\mathrm{KD}$ enhances mitochondrial functioning and biogenesis (Bough et al., 2006; Maalouf et al., 2009; Kim do et al., 2010), it is logical to ask whether patients with known mitochondrial cytopathies might derive a benefit from the KD and/or ketone bodies such as $\mathrm{BHB}$. At the same time, it must be considered that inherent mitochondrial dysfunction might predispose individuals to adverse toxicities from high fatty acid loads that could overwhelm $\beta$-oxidation within the mitochondrial matrix. Experimental data described above attest to significant improvements in mitochondrial function, and many lines of evidence point to the rationale of therapeutically targeting mitochondrial bioenergetics for other disease states (Wallace et al., 2010), but is there any clinical evidence in patients with intrinsic mitochondrial disorders? Kang et al. (2007) reported that the KD was both safe and effective in 14 pediatric patients with established mitochondrial defects in complexes I, II, and IV, all of whom had medically intractable epilepsy. These authors observed that half of these patients became seizure-free on the $\mathrm{KD}$, and only four patients failed to respond. Hence, these preliminary data suggest that the KD is not necessarily contraindicated in patients with mitochondrial respiratory chain abnormalities. However, KD treatment is not recommended in individuals with primary carnitine deficiencies [including mutations in carnitine palmitoyl transferase (CPT) I or II and mitochondrial translocase] and fatty acid $\beta$-oxidation abnormalities (e.g., medium-chain acyl dehydrogenase deficiency; Kossoff et al., 
2009). Thus, it is critical to determine the specific mitochondrial defect when considering treatment with the $\mathrm{KD}$, to avert clinical deterioration.

\section{THE KD IN BRAIN TRAUMA}

Unfortunately, the incidence of brain injury is increasing in both civilian and military contexts. Brain injury, either due to a penetrating injury or to blunt/blast trauma, can lead to severe cognitive and motor consequences. Further, the occurrence of epilepsy months to years following brain trauma adds to the morbidity of affected individuals, and speaks to the emergence of hyperexcitable neuronal circuits over time. Hence, in light of the clinical problem of post-traumatic epileptogenesis and the fact that the KD can reduce seizure activity, the notion has emerged that dietary therapy might ameliorate brain injury and possibly, long-term consequences such as epilepsy.

Several recent animal studies support this idea, and investigators have principally focused on ketone bodies (Prins, 2008a). Using a controlled cortical impact (CCI) injury model, Prins et al. (2005) showed that pre-treatment with a KD significantly reduced cortical contusion volume in an age-related manner that correlated with maturation-dependent differences in cerebral metabolism and ketone utilization. Later, they showed that cognitive and motor functioning was also improved with $\mathrm{KD}$ treatment (Appelberg et al., 2009). Further, using a weight drop model, Hu et al. (2009) showed that the KD pre-treatment reduced Bcl-2 (also known as Bax) mRNA and protein levels $72 \mathrm{~h}$ after trauma, indicating that apoptotic neurodegeneration could be prevented with this diet. Consistent with these observations, it was found that fasting - which shares the key feature of ketosis with the $\mathrm{KD}$ - led to significant tissue sparing in brain following CCI injury, and that again ketosis (with improved mitochondrial functioning) rather than the relative hypoglycemia seen with fasting was the important determinant of neuroprotection (Davis et al., 2008).

With respect to anti-epileptogenesis following head injury, the data regarding KD effects are mixed. KD treatment - either before or after fluid percussion injury in rats - did not alter later seizure sensitivity to fluorothyl, even though the degree of hippocampal cell loss was reduced by pre- but not post-treatment (Schwartzkroin et al., 2010). Similarly, in the lithium-pilocarpine model of temporal lobe epilepsy, KD treatment prior to induction led to morphological neuroprotection in the hippocampus but did not affect latency to onset of spontaneous recurrent seizures (Linard et al., 2010). In contrast, Jiang et al. (2012) recently reported that the $\mathrm{KD}$ increased after-discharge thresholds and reduced generalized seizure occurrence in a rat amygdala kindling model. Thus, at this juncture, there is no consensus regarding whether the KD is anti-epileptogenic following a variety of traumatic insults and manipulations. However, given the recent finding that the KD inhibits the mammalian target of rapamycin (mTOR) pathway (McDaniel et al., 2011), which has been linked to modulation of epileptogenesis (McDaniel and Wong, 2011), further studies in different animal models are clearly warranted. What is unambiguous, nevertheless, is the age-dependence of the effects of the KD in ameliorating the consequences of head injury (Prins, 2008b; Deng-Bryant et al., 2011).

\section{THE KD IN PSYCHIATRIC DISORDERS (DEPRESSION)}

Mood stabilizing properties of the KD have been hypothesized (El-Mallakh and Paskitti, 2001), but no clinical studies have been conducted as of this writing. The potential role of the KD in depression has been studied in the forced choice model of depression in rats, which led to a beneficial effect similar to that afforded by conventional antidepressants (Murphy et al., 2004; Murphy and Burnham, 2006).

\section{THE KD IN AUTISM}

Autism is a neurodevelopmental disorder that affects language development and social function. The heterogeneous etiologies leading to autism spectrum disorders, plus the uncertainty about what causes autism in the majority of "idiopathic" cases, has hampered the development of a universally beneficial treatment, aside from symptomatic treatment of autism-related behaviors such as aggression or anxiety. Now, limited clinical evidence raises the intriguing possibility that the KD might be helpful to alleviate some of the abnormal behaviors seen in children with autism spectrum disorders. Using a KD variant consisting of MCT, 10 of 18 autistic children demonstrated moderate or significant behavioral improvement (by a blinded rater) after a 6-month trial of providing the diet for 4 weeks of $\mathrm{KD}$ diet treatment alternating with 2 weeks of normal diet, in 6-week cycles (Evangeliou et al., 2003). This study was carried out on the island of Crete, where the frequency of autism is high but the possibility of genetic inbreeding is also significant. Therefore, these findings need to be interpreted cautiously and larger longitudinal studies are needed. The potential involvement of adenosine, an endogenous neuromodulator and anticonvulsant, in ameliorating autistic behaviors raises the possibility of overlap with KD mechanisms (Masino et al., 2011). As a caveat, many children with autism poorly tolerate changes in dietary and other routines, which could impact implementation of dietary therapies, which require strict adherence.

\section{THE KD IN MIGRAINE}

Migraine is a paroxysmal neurological disorder having considerable clinical phenotypic overlap with epilepsy (Rogawski, 2008). Although the intrinsic mechanisms underlying seizures and migraine attacks differ in many fundamental respects, there are theoretical reasons to consider the KD for chronic migraine. Both disorders involve paroxysmal excitability changes in the brain, and there is considerable overlap in the array of pharmacological agents used to treat these conditions. Although it might seem unlikely that an individual with migraine would undertake such a complicated dietary regimen as the $\mathrm{KD}$, in light of suboptimal alternatives, this choice is worthy of consideration, particularly in the medically refractory population (Maggioni et al., 2011).

Interestingly, the first report of using the KD for migraine came in 1928, only a few years after the diet's first use for epilepsy (Schnabel, 1928). Nine of 28 patients reported "some improvement," although the validity of this clinical study is uncertain and some patients admitted poor compliance. Compliance might be better with the less restrictive modified Atkins diet, which has also shown promise for migraine treatment (Kossoff et al., 2010). Other case reports exist but there are no large clinical series or trials. Notwithstanding this limitation, laboratory investigations 
have found that both short-term and long-term treatment with either MCT or long-chain triglyceride forms of the KD resulted in a significant reduction in the velocity of cortical spreading depression (CSD) velocity in immature rats (de Almeida Rabello Oliveira et al., 2008). Another intriguing aspect of this study was the observation that triheptanoin - an anaplerotic substrate that enhances tricarboxylic acid cycle function - had a notable effect in retarding CSD, consistent with a later report that triheptanoin supplementation raised pentylenetetrazol tonic seizure threshold and delayed the development of corneal kindled seizures (Willis et al., 2010).

\section{SUMMARY}

Despite the relative lack of clinical data, there is an emerging literature supporting the broad use of the $\mathrm{KD}$ (and its variants)

\section{REFERENCES}

Acharya, M. M., Hattiangady, B., and Shetty, A. K. (2008). Progress in neuroprotective startegies for preventing epilepsy. Prog. Neurobiol. 84, 363-404.

Aft, R. L., Zhang, F. W., and Gius, D. (2002). Evaluation of 2-deoxy-Dglucose as a chemotherapeutic agent: mechanism of cell death. Br. J. Cancer 87, 805-812.

Appelberg, K. S., Hovda, D. A., and Prins, M. L. (2009). The effects of a ketogenic diet on behavioral outcome after controlled cortical impact injury in the juvenile and adult rat. J. Neurotrauma 26, 497-506.

Balietti, M., Casoli, T., DiStefano, G., Giorgetti, B., Aicardi, G., and Fattoretti, P. (2010a). Ketogenic diets: an historical antiepieptic therapy with promising potentialities for the aging brain. Ageing Res. Rev. 9, 273-279.

Balietti, M., Giorgetti, B., DiStefano, G., Casoli, T., Platano, D., Solazzi, M., Bertoni-Freddari, C., Aicardi, G., Lattanzio, F., and Fattoretti, P. (2010b). A ketogenic diet increases succinic dehydrogenase $(\mathrm{SDH})$ activity and recovers age-related decrease in numeric density of SDH-positive mitochondria in cerebellar Purkinje cells of lateadult rats. Micron 41, 143-148.

Balietti, M., Giorgetti, B., Fattoretti, P., Grossi, Y., DiStefano, G., Casoli, T., Platano, D., Solazzi, M., Orlando, F., Aicardi, G., and Bertoni-Freddari, C. (2008). Ketogenic diets cause opposing changes in synaptic morphology in CA1 hippocampus and dentate gyrus of late-adult rats. Rejuvenation Res. 11, 631-640.

Baranano, K. M., and Hartman, A. L. (2008). The ketogenic diet: uses in epilepsy and other neurologic illnesses. Curr. Treat. Options Neurol. 10, 410-419.

Bough, K. J., and Stafstrom, C. E. (2010). "The ketogenic diet: scientific principles underlying it use," in Epilepsy: Mechanisms, Models, and Translational Perspectives, eds J. M. Rho, R. Sankar, and C. E. Stafstrom (Boca Raton, FL: CRC Press), 417-439.

Bough, K. J., Wetherington, J., Hassel, B., Pare, J. F., Gawryluk, J. W., Greene, J. G., Shaw, R., Smith, Y., Geiger, J. D., and Dingledine, R. J. (2006). Mitochondrial biogenesis in the anticonvulsant mechanism of the ketogenic diet. Ann. Neurol. 60, 223-235.

Cardinali, S., Canafoglia, L., Bertoli, S., Franceschetti, S., Lanzi, G., Tagliabue, A., and Veggiotti, P. (2006). A pilot study of a ketogenic diet in patients with Lafora body disease. Epilepsy Res. 69, 129-134.

Chu-Shore, C. J., Major, P., Camposano, S., Muzykewicz, D., and Thiele, E. A. (2010). The natural history of epilepsy in tuberous sclerosis complex. Epilepsia 51, 1236-1241.

Contestabile, A. (2009). Benefits of caloric restriction on brain aging and related pathological states: understanding mechanisms to devise novel therapies. Curr. Med. Chem. 16, 350-361.

Cullingford, T. (2008). Peroxisome proliferator-activated receptor alpha and the ketogenic diet. Epilepsia 49(Suppl. 8), 70-72.

Davis, L. M., Pauly, J. R., Readnower, R. D., Rho, J. M., and Sullivan, P. G. (2008). Fasting is neuroprotective following traumatic brain injury. $J$. Neurosci. Res. 86, 1812-1822.

de Almeida Rabello Oliveira, M., da Rocha Ataíde, T., de Oliveira, S. L., de Melo Lucena, A. L., de Lira, C. E., Soares, A. A., de Almeida,

against a variety of neurological conditions. These preliminary studies are largely based on the fundamental idea that metabolic shifts may lead to neuroprotective actions (Gasior et al., 2006; Maalouf et al., 2009). How can a simple dietary change lead to improvement in disorders with such a huge span of pathophysiological mechanisms? Alterations in energy metabolism appear to be a common theme. So while the mechanisms through which the KD exerts such effects are likely diverse (Maalouf et al., 2009; Rho and Stafstrom, 2011), there may indeed be one or more common final pathways that are mechanistically shared. Ultimately, the details of how that altered metabolism reduces neuronal excitability, abrogates ongoing neurodegeneration, or mitigates functional disability remain unknown. Herein lay rich opportunities for further investigation, in both the laboratory and the clinic, in the broad realm of translational neurosciences.

C. B., Ximenes da Silva, A. (2008). Effects of short-term and long-term treatment with medium- and longchain triglycerides ketogenic diet on cortical spreading depression in young rats. Neurosci. Lett. 434, 66-70.

Deng-Bryant, Y., Prins, M. L., Hovda, D. A., and Harris, N. G. (2011) Ketogenic diet prevents alterations in brain metabolism in young but not adult rats after traumatic brain injury. J. Neurotrauma 28 , 1813-1825.

El-Mallakh, R. S., and Paskitti, M. E. (2001). The ketogenic diet may have mood-stabilizing properties. Med. Hypotheses 57, 724-726.

Evangeliou, A., Vlachonikolis, I., Mihailidou, H., Spilioti, M., Skarpalezou, A., Makaronas, N., Prokopiou, A., Christodoulou, P., Liapi-Adamidou, G., Helidonis, E., Sbyrakis, S., and Smeitink, J. (2003). Application of a ketogenic diet in children with autistic behavior: pilot study. J. Child Neurol. 18, 113-118.

Freeman, J. M., Kossoff, E. H., Freeman, J. B., and Kelly, M. T. (2007). The Ketogenic Diet - A Treatment for Children and Others with Epilepsy. New York: Demos Publications.

Freeman, J. M., Vining, E. P., Kossoff, E. H., Pyzik, P. L., Ye, X., and Goodman, S. N. (2009). A blinded, crossover study of the efficacy of the ketogenic diet. Epilepsia 50, 322-323.

Freemantle, E., Vandal, M., TremblayMercier, J., Plourde, M., Poirier, J., and Cunnane, S. C. (2009). Metabolic response to a ketogenic breakfast in the healthy elderly. J. Nutr. Health Aging 13, 293-298.

Garriga-Canut, M., Schoenike, B., Qazi, R., Bergendahl, K., Daley, T. J., Pfender, R. M., Morrison, J. F., Ockuly, J., Stafstrom, C., Sutula, T. and Roopra, A. (2006). 2-Deoxy-Dglucose reduces epilepsy progression by NRSF-CtBP-dependent metabolic regulation of chromatin structure. Nat. Neurosci. 9, 1382-1387.

Gasior, M., Rogawski, M. A., and Hartman, A. L. (2006). Neuroprotective and disease-modifying effects of the ketogenic diet. Behav. Pharmacol. 17, 431-439.

Grabacka, M., Pierzchalska, M., and Reiss, K. (2010). Peroxisome proliferator activated receptor $\alpha$ ligands as anti-cancer drugs targeting mitochondrial metabolism. Curr. Pharm. Biotechnol. PMID: 21133850.

Greene, A. E., Todorova, M. T., McGowan, R., and Seyfried, T. N. (2001). Caloric restriction inhibits seizure susceptibility in epileptic EL mice by reducing blood glucose. Epilepsia 42, 1371-1378.

$\mathrm{Gu}$, Y., Luchsinger, J. A., Stern, Y., and Scarmeas, N. (2010). Mediterranean diet, inflammatory and metabolic biomarkers, and risk of Alzheimer's disease. J. Alzheimers Dis. 22, 483-492.

Hartman, A. L., Gasior, M., Vining, E. P. G., and Rogawski, M. A. (2007). The neuropharmacology of the ketogenic diet. Pediatr. Neurol. 36, 281-292.

Henderson, S. T., Vogel, J. L., Barr, L. J., Garvin, F., Jones, J. J., and Costantini, L. C. (2009). Study of the ketogenic agent AC-1202 in mild to moderate Alzheimer's disease: a randomized, double-blind, placebocontrolled, multicenter trial. Nutr. Metab. (Lond.) 6, 31.

Hu, Z. G., Wang, H. D., Qiao, L., Yan, W., Tan, Q. F., and Yin, H. X. (2009). The protective effect of the ketogenic diet on traumatic brain injury-induced cell death in juvenile rats. Brain Inj. 23, 459-465. 
Jeong, E. A., Jeon, B. T., Shin, H. J., Kim, N., Lee, D. H., Kim, H. J., Kang, S. S., Cho, G. J., Choi, W. S., and Roh, G. S. (2011). Ketogenic diet-induced peroxisome proliferator-activated receptor- $\gamma$ activation decreases neuroinflammation in the mouse hippocampus after kainic acidinduced seizures. Exp. Neurol. 232, 195-202.

Jiang, Y., Yang, Y., Wang, S., Ding, Y., Guo, Y., Zhang, M. M., Wen, S. Q., and Ding, M. P. (2012). Ketogenic diet protects against epileptogenesis as well as neuronal loss in amygdaloid-kindling seizures. $\mathrm{Neu}$ rosci. Lett. 508, 22-26.

Kang, H. C., Lee, Y. M., Kim, H. D., Lee, J. S., and Slama, A. (2007). Safe and effective use of the ketogenic diet in children with epilepsy and mitochondrial respiratory chain complex defects. Epilepsia 48, 82-88.

Kapogiannis, D., and Mattson, M. P. (2011). Disrupted energy metabolism and neuronal circuit dysfunction in cognitive impairment and Alzheimer's disease. Lancet Neurol. 10, 187-198.

Kashiwaya, Y., Takeshima, T., Mori, N., Nakashima, K., Clarke, K., and Veech, R. L. (2000). D-betahydroxybutyrate protects neurons in models of Alzheimer's and Parkinson's disease. Proc. Natl. Acad. Sci. U.S.A. 97, 5440-5444.

Kemnitz, J. W. (2011). Calorie restriction and aging in nonhuman primates. ILAR J. 52, 66-77.

Kessler, S. K., Neal, E. G., Camfield, C. S., and Kossoff, E. H. (2011). Dietary therapies for epilepsy: future research. Epilepsy Behav. 22, 17-22.

Kim do, Y., Davis, L. M., Sullivan, P. G., Maalouf, M., Simeone, T. A., vanBrederode, J., and Rho, J. M. (2007). Ketone bodies are protective against oxidative stress in neocortical neurons. J. Neurochem. 101, 1316-1326.

Kim do, Y., and Rho, J. M. (2008). The ketogenic diet and epilepsy. Curr. Opin. Clin. Nutr. Metab. Care 11, 113-120.

Kim do, Y., Vallejo, J., and Rho, J. M. (2010). Ketones prevent synaptic dysfunction induced by mitochondrial respiratory complex inhibitors. J. Neurochem. 114, 130-141.

Kossoff, E. H., Huffman, J., Turner, Z., and Gladstein, J. (2010). Use of the modified Atkins diet for adolescents with chronic daily headache. Cephalalgia 30, 1014-1016.

Kossoff, E. H., and McGrogan, J. R. (2005). Worldwide use of the ketogenic diet. Epilepsia 46, 280-289.

Kossoff, E. H., Zupec-Kania, B. A., Amark, P. E., Ballaban-Gil, K. R.,
Bergqvist, C. A. G., Blackford, R., Buchhalter, J. R., Caraballo, R. H., Cross, H. J., Dahlin, M. G., Donner, E. J., Klepper, J., Jehle, R. S., Kim, H. D., Liu, C. Y. M., Nation, J., Nordli, D. R. Jr., Pfeifer, H. H., Rho, J. M., Stafstrom, C. E., Thiele, E. A., Turner, Z., Wirrell, E. C., Wheless, J. W., Veggiotti, P., and Vining, E. P. (2009). Optimal clinical management of children receiving the ketogenic diet: recommendations of the International Ketogenic Diet Study Group. Epilepsia 50, 304-317.

Linard, B., Ferrandon, A., Koning, E., Nehlig, A., and Raffo, E. (2010). Ketogenic diet exhibits neuroprotective effects in hippocampus but fails to prevent epileptogenesis in the lithium-pilocarpine model of mesial temporal lobe epilepsy in adult rats. Epilepsia 51, 1829-1836.

Ma, W., Berg, J., and Yellen, G. (2007). Ketogenic diet metabolites reduce firing in central neurons by opening K(ATP) channels. J. Neurosci. 27, 3618-3625.

Maalouf, M., Rho, J. M., and Mattson, M. P. (2009). The neuroprotective properties of calorie restriction, the ketogenic diet, and ketone bodies. Brain Res. Rev. 59, 293-315.

Maggioni, F., Margoni, M., and Zanchin, G. (2011). Ketogenic diet in migraine treatment: a brief but ancient history. Cephalalgia 31, 1150-1151.

Masino, S. A., and Geiger, J. D. (2008). Are purines mediators of the anticonvulsant/neuroprotective effects of ketogenic diets? Trends Neurosci. 31, 273-278.

Masino, S. A., Kawamura, M. Jr., Plotkin, L. M., Svedova, J., DiMario, F. J. Jr., and Eigsti, I. M. (2011). The relationship between the neuromodulator adenosine and behavioral symptoms of autism. Neurosci. Lett. 500, $1-5$.

McDaniel, S. S., Rensing, N. R., Thio, L. L., Yamada, K. A., and Wong, M. (2011). The ketogenic diet inhibits the mammalian target of rapamycin (mTOR) pathway. Epilepsia 52, e7el1.

McDaniel, S. S., and Wong, M. (2011). Therapeutic role of mammalian target of rapamycin (mTOR) inhibition in preventing epileptogenesis. Neurosci. Lett. 497, 231-239.

Murphy, P., and Burnham, W. M. (2006). The ketogenic diet causes a reversible decrease in activity level in Long-Evans rats. Exp. Neurol. 201, 84-89.

Murphy, P., Likhodii, S., Nylen, K., and Burnham, W. M. (2004). The antidepressant properties of the ketogenic diet. Biol. Psychiatry 56, 981-983.

Neal, E. G., Chaffe, H., Schwartz, R. H., Lawson, M. S., Edwards, N., Fitzsimmons, G., Whitney, A., and Cross, J. H. (2008). The ketogenic diet for the treatment of childhood epilepsy: a randomised controlled trial. Lancet Neurol. 7, 500-506.

Nebeling, L. C., Miraldi, F., Shurin, S. B., and Lerner, E. (1995). Effects of a ketogenic diet on tumor metabolism and nutritional status in pediatric oncology patients: two case reports. J. Am. Coll. Nutr. 14, 202-208.

Nehlig, A. (1999). Age-dependent pathways of brain energy metabolism: the suckling rat, a natural model of the ketogenic diet. Epilepsy Res. 37, 211-221.

Noebels, J. L. (2011). A perfect storm: converging paths of epilepsy and Alzheimer's dementia intersect in the hippocampal formation. Epilepsia 52(Suppl. 1), 39-46.

Otto, C., Kaemmerer, U., Illert, B. Muehling, B., Pfetzer, N., Wittig, R., Voelker, H. U., Thiede, A., and Coy, J. F. (2008). Growth of human gastric cancer cells in nude mice is delayed by a ketogenic diet supplemented with omega3 fatty acids and medium-chain triglycerides. BMC Cancer 8, 122. doi:10.1186/1471-2407-8-122

Palop, J. J., Chin, J., Roberson, E. D., Wang, J., Thwin, M. T., Bien-Ly, N., Yoo, J., Ho, K. O., Yu, G. Q., Kreitzer, A., Finkbeiner, S., Noebels, J. L., and Mucke, L. (2007). Aberrant excitatory neuronal activity and compensatory remodeling of inhibitory hippocampal circuits in mouse models of Alzheimer's disease. Neuron 55, 697-711.

Palop, J. J., and Mucke, L. (2009). Epilepsy and cognitive impairments in Alzheimer disease. Arch. Neurol. 66, 435-440.

Pelicano, H., Martin, D. S., Xu, R.H., and Huang, P. (2006). Glycolysis inhibition for anticancer treatment. Oncogene 25, 4633-4646.

Prins, M. L. (2008a). Cerebral metabolic adaptation and ketone metabolism after brain injury. J. Cereb. Blood Flow Metab. 28, 1-16.

Prins, M. L. (2008b). Diet, ketones, and neurotrauma. Epilepsia 49(Suppl. 8), 111-113.

Prins, M. L., Fujima, L. S., and Hovda, D. A. (2005). Age-dependent reduction of cortical contusion volume by ketones after traumatic brain injury. J. Neurosci. Res. 82, 413-420.

Puchowicz, M. A., Zechel, J. L., Valerio, J., Emancipator, D. S., Xu, K.,
Pundik, S., LaManna, J. C., and Lust, W. D. (2008). Neuroprotection in diet-induced ketotic rat brain after focal ischemia. J. Cereb. Blood Flow Metab. 28, 1907-1916.

Redman, L. M., and Ravussin, E. (2011). Caloric restriction in humans: impact on physiological, psychological, and behavioral outcomes. Antioxid. Redox Signal. 14, 275-278.

Rho, J. M., and Stafstrom, C. E. (2011). The ketogenic diet: what has science taught us? Epilepsy Res. doi:10.1016/j.eplepsyres.2011.05.021 Roberson, E. D., Halabisky, B., Yoo, J. W., Yao, J., Chin, J., Yanm, F., Wu, T., Hamto, P., Devidze, N., Yu, G. Q., Palop, J. J., Noebels, J. L., and Mucke, L. (2011). Amyloid- $\beta /$ Fyninduced synaptic, network, and cognitive impairments depend on tau levels in multiple mouse models of Alzheimer's disease. J. Neurosci. 31, 700-711.

Rogawski, M. A. (2008). Common pathophysiologic mechanisms in migraine and epilepsy. Arch. Neurol. 65, 709-714.

Roth, J., Szulc, A. L., and Danoff, A. (2011). Energy, evolution, and human diseases: an overview. Am. J. Clin. Nutr. 93, 875S-883S.

Schiff, M., Bénit, P., Coulibaly, A., Loublier, S., El-Khoury, R., and Rustin, P. (2011). Mitochondrial response to controlled nutrition in health and disease. Nutr. Rev. 69, 65-75.

Schmidt, M., Pfetzer, N., Schwab, M., Strauss, I., and Kämmerer, U. (2011). Effects of a ketogenic diet on the quality of life in 16 patients with advanced cancer: a pilot trial. Nutr. Metab. (Lond.) 8, 54.

Schnabel, T. G. (1928). An experience with a ketogenic diet in migraine. Ann. Int. Med. 2, 341-347.

Schwartzkroin, P. A., Wenzel, H. J., Lyeth, B. G., Poon, C. C., Delance, A. Van, K. C., Campos, L., and Nguyen, D. V. (2010). Does ketogenic diet alter seizure sensitivity and cell loss following fluid percussion injury? Epilepsy Res. 92, 74-84.

Seyfried, T. N., Marsh, J., Shelton, L. M., Huysentruyt, L. C., and Mukherjee, P. (2011). Is the restricted ketogenic diet a viable alternative to the standard of care for managing malignant brain cancer? Epilepsy Res. [Epub ahead of print].

Siva, N. (2006). Can ketogenic diet slow progression of ALS? Lancet Neurol. 5, 476.

Srikanth, V., Maczurek, A., Phan, T., Steele, M., Westcott, B., Juskiw, D., and Münch, G. (2011). Advanced glycation endproducts and their 
receptor RAGE in Alzheimer's disease. Neurobiol. Aging 32, 763-777.

Stafford, P., Abdelwahab, M. G., Kimdo, Y., Preul, M. C., Rho, J. M., and Scheck, A. C. (2010). The ketogenic diet reverses gene expression patterns and reduces reactive oxygen species levels when used as an adjuvant therapy for glioma. Nutr. Metab. (Lond.) 7, 74.

Stafstrom, C. E. (2004). Dietary approaches to epilepsy treatment: old and new options on the menu. Epilepsy Curr. 4, 215-222.

Tai, K. K., Nguyen, N., Pham, L., and Truong, D. D. (2008). Ketogenic diet prevents cardiac arrestinduced cerebral ischemic neurodegeneration. J. Neural Transm. 115, 1011-1017.

Tai, K. K., Pham, L., and Truong, D. D. (2009). Intracisternal administration of glibenclamide or 5hydroxydecanoate does not reverse the neuroprotective effect of ketogenic diet against ischemic brain injury-induced neurodegeneration. Brain Inj. 23, 1081-1088.

VanderAuwera, I., Wera, S., VanLeuven, F., and Henderson, S. T. (2005). A ketogenic diet reduces amyloid beta 40 and 42 in a mouse model of Alzheimer's disease. Nutr. Metab. (Lond.) 2, 28.

Vanitallie, T. B., Nonas, C., DiRocco, A., Boyar, K., Hyams, K., and
Heymsfield, S. B. (2005). Treatment of Parkinson disease with dietinduced hyperketonemia: a feasibility study. Neurology 64, 728-730.

Vining, E., Freeman, J., Ballaban-Gil, K., Camfield, C., Camfield, P., Holmes, G., Shinnar, S., Shuman, R., Trevathan, E., and Wheless, J. (1998). A multicenter study of the efficacy of the ketogenic diet. Arch. Neurol. 55, 1433-1437.

Vining, E. P. G. (1999). Clinical efficacy of the ketogenic diet. Epilepsy Res. 37, 181-190.

Voskuyl, R. A., and Vreugdenhil, M. (2001). "Effects of essential fatty acids on voltage-regulated ionic channels and seizure thresholds in animals," in Fatty Acids: Physiological and Behavioral Functions, eds D. Mostofsky, S. Yehuda, and N. Jr. Salem (Totowa, NJ: Humana Press), 63-78.

Waldbaum, S., and Patel, M. (2010). Mitochondrial dysfunction and oxidative stress: a contributing link to acquired epilepsy? J. Bioenerg. Biomembr. 42, 449-455.

Wallace, D. C., Fan, W., and Procaccio, V. (2010). Mitochondrial energetics and therapeutics. Annu. Rev. Pathol. 5, 297-348.

Wheless, J. W. (2008). History of the ketogenic diet. Epilepsia 49(Suppl. 8), 3-5.
Willis, S., Stoll, J., Sweetman, L., and Borges, K. (2010). Anticonvulsant effects of a triheptanoin diet in two mouse chronic seizure models. Neurobiol. Dis. 40, 565-572.

Xu, K., Sun, X., Eroku, B. O., Tsipis, C. P., Puchowicz, M. A., and LaManna, J. C. (2010). Diet-induced ketosis improves cognitive performance in aged rats. Adv. Exp. Med. Biol. 662, 71-75.

Yamada, K. A. (2008). Calorie restriction and glucose regulation. Epilepsia 49(Suppl. 8), 94-96.

Yang, X., and Cheng, B. (2010). Neuroprotective and anti-inflammatory activities of ketogenic diet on MPTPinduced neurotoxicity. J. Mol. Neurosci. 42, 145-153.

Yao, J., Chen, S., Mao, Z., Cadenas, E., and Brinton, R. D. (2011). 2Deoxy-D-glucose treatment induces ketogenesis, sustains mitochondrial function, and reduces pathology in female mouse model of Alzheimer's disease. PLoS ONE 6, e21788. doi:10.1371/journal.pone.0021788

Zhao, Z., Lange, D. J., Voustianiouk, A., MacGrogan, D., Ho, L. Suh, J., Humala, N., Thiyagarajan, M., Wang, J., and Pasinetti, G. M. (2006). A ketogenic diet as a potential novel therapeutic intervention in amyotrophic lateral sclerosis. BMC Neurosci. 7, 29. doi:10.1186/1471-2202-7-29
Zuccoli, G., Marcello, N., Pisanello, A., Servadei, F., Vaccaro, S., Mukherjee, P., and Seyfried, T. N. (2010). Metabolic management of glioblastoma multiforme using standard therapy together with a restricted ketogenic diet: case report. Nutr. Metab. (Lond.) 7, 33.

Conflict of Interest Statement: The authors declare that the research was conducted in the absence of any commercial or financial relationships that could be construed as a potential conflict of interest.

Received: 17 January 2012; paper pending published: 25 January 2012; accepted 21 March 2012; published online: 09 April 2012.

Citation: Stafstrom CE and Rho JM (2012) The ketogenic diet as a treatment paradigm for diverse neurological disorders. Front. Pharmacol. 3:59. doi: 10.3389/fphar.2012.00059

This article was submitted to Frontiers in Neuropharmacology, a specialty of Frontiers in Pharmacology.

Copyright (c) 2012 Stafstrom and Rho. This is an open-access article distributed under the terms of the Creative Commons Attribution Non Commercial License, which permits non-commercial use, distribution, and reproduction in other forums, provided the original authors and source are credited. 